\title{
Consumo de produtos tecnológicos: da funcionalidade ao status
}

\author{
Consumption of technological products: from functionality to \\ status
}

Lucas Emmanuel Nascimento Silva - lucasemmanuel1998@gmail.com

Manoel Bastos Gomes Neto - neto26bastos@gmail.com

Rebeca da Rocha Grangeiro - rebeca.grangeiro@ufca.edu.br

Jailson Santana Carneiro - jailson192@gmail.com

\section{RESUMO}

Este artigo tem como objetivo determinar a relação entre consumo de status e grau de adoção de inovação. Diante disso, foam utilizadas a Escala de Domínio-Específico de Inovatividade (1991) e a Escala de Consumo de Status (1999), esta última dividida em três construtos: sociabilidade, consumo de status e não funcionalidade. A pesquisa foi conduzida através de questionários. A amostra é composta por 822 estudantes de escolas e universidades públicas e privadas. Nos resultados, apesar da inovatividade impactar positivamente nas dimensões do consumo de status, a variação do consumo por status de objetos tecnológicos é pouco explicada a partir do comportamento inovativo. Ademais, os resultados indicam que: 1) consumidores com alto grau de inovatividade são altamente integrados com grupos sociais; 2) estes consumidores são mais preocupados com as características simbólicas dos bens; 3) estes consumidores identificam nos novos produtos tecnológicos uma forma de expressar status para seu arredor.

Palavras-chave: Consumo de Status; Inovatividade; Consumo de Produtos Tecnológicos; Comportamento do Consumidor; Consumo Simbólico.

\section{ABSTRACT}

This article aims at determining the relationship between status consumption and the degree of innovation adoption. For this goal, we used the DomainSpecific Innovativeness Scale (1991) and the Status Consumption Scale (1999), the latter is divided into three constructs: sociability, status consumption and nonfunctional. The survey was conducted through a questionnaire. The sample was composed of 822 students from public and 
private high schools and colleges. In the results, even with the positive relationship between innovativeness and status consumption, the variation on the status consumption explained by the innovativeness is not highly explained. The results indicate that: 1) innovative consumers are highly integrated with social groups; 2) innovative consumers are more concerned about the symbolic aspects of the goods; 3) innovative consumers seek in new technological products a way of obtaining and showing status for their surroundings.

Keywords: Status Consumption; Innovativeness; Consumption of Technological Products; Consumer Behavior; Symbolic Consumption.

\section{INTRODUÇÃO}

As teorias sobre o comportamento do consumidor nos estudos do marketing têm se modificado ao longo dos anos com o intuito de melhorar a compreensão das características intrínsecas e extrínsecas que influenciam as decisões dos indivíduos. As transformações ocorridas no campo econômico, social e tecnológico fizeram emergir um novo pensamento sobre a sociedade de consumo, modificando a visão de consumo exclusivamente para satisfação de necessidade e ampliando a visão do consumo a partir de seu potencial para a construção de identidade (Campbell, 2006).

Na perspectiva de construção de identidade, uma vertente do comportamento de consumo recorrente nos estudos de marketing é o consumo de luxo (Eastman \& Eastman, 2015). Pesquisas recentes buscaram identificar novas perspectivas e motivações para o consumo de luxo a exemplo do consumo de luxo realizado por mulheres e a expansão do self na experiência da marca de luxo (eg. Semaan et al., 2019, Kerviler, Rodriguez, 2019).

O consumo de status pode ser compreendido como o interesse que os indivíduos possuem de melhorar sua posição social através do consumo de produtos que simbolizam status para pessoas significantes (Eastman \& Eastman, 2015). Pereira et al., (2018) testararam o modelo e motivações de consumo de status proposto por Eastman e Eastman (2015) e identificaram a relevância dos fatores internos (o hedonismo, o perfeccionismo e a recompensa pessoa) e externos (o efeito Veblen, o efeito esnobe e o efeito 
adesão). As motivações internas e externas para o consumo de produtos luxuosos são independentes e as motivações externas possuem maior influência neste consumo (Pereira et al., 2018).

Diante desta perspectiva, no consumo de status, o indivíduo passa a consumir com o objetivo principal de obter reconhecimento de seu grupo social ou do qual deseja pertencer. Estudos recentes identificaram que essas motivações por prestígio social estão influenciando a compra de novos produtos (Costa \& Arruda Filho, 2016). Diante disso, é importante investigar em quais cenários as necessidades dos consumidores que buscam prestígio através do consumo de novidades tecnológicas são atendidas (Costa \& Arruda Filho, 2016).

O mercado tecnológico está em constante processo de mudança, tornando-se progressivamente mais qualificado para moldar o comportamento dos consumidores diante das novidades mercadológicas, uma vez que estes novos produtos, quando bem difundidos e experimentados provocam diversas mudanças sociais na vida dos indivíduos (Barboza \& Arruda Filho, 2012).

O self estendido que mostra sinais para outras pessoas formarem impressões sobre nós, acabou sendo potencializado por objetos tecnológicos (Belk, 2013). A mudança na visão dos consumidores de buscarem produtos luxuosos em detrimento de produtos comuns foi afetada pelo desejo de construir e presentear o self.

Considerando que os produtos tecnológicos possuem um ciclo de inovação constante e normalmente possuem um preço alto, tornando-os produtos desejáveis e pouco acessíveis, estes produtos são possuidores de status (Strehlau \& Aranha, 2004). Além disso, a determinação do produto possuir ou não status depende da percepção das pessoas, da cultura e da sociedade (Bilge, 2015). 
Especialmente no âmbito do grupo formado por jovens, que inclui os adolescentes, esses produtos tecnológicos ganham destaque, pois atendem as expectativas individuais e as dos grupos de referência, em especial dos amigos. A necessidade de pertencimento acaba fazendo que os hábitos de consumo resultem em escape nesta fase da vida (Peixoto et al., 2018).

Dito disto, este artigo tem como objetivo analisar a relação entre o grau de adoção de produtos tecnológicos inovadores e o consumo de status, identificando como o consumo de status pode ser influenciado pela propensão do indivíduo de adotar uma inovação mais cedo que outros do seu grupo social.

Desta forma, o presente artigo pretende colaborar com as teorias da área do marketing: consumo de status; comportamento de jovens consumidores; e consumo de produtos tecnológicos. Além disso, o estudo contribui na compreensão do perfil e das necessidades de jovens consumidores, para melhor satisfazê-las.

\section{ESTRUTURA TEÓRICA E DESENVOLVIMENTO DE HIPÓTESES}

A estrutura teórica está dividida em duas seções. Na primeira são apresentados os aspectos da teoria do consumo de status e na segunda são discutidos o consumo de inovação e a mensuração da tendência para a adoção de inovação. Ademais, são estabelecidos as relações entre os construtos a serem abordados, e indicando as hipóteses que serão testadas pela pesquisa.

\section{Consumo e a obtenção de status}

O consumo é um construtor de identidade, quando consumimos, mostramos a forma como vemos o mundo, além de proporcionar um sentimento de pertencimento e conceber a construção de laços sociais a um determinado grupo (Cortez, 2009). 
Existem três motivos que levam os indivíduos ao consumo (Bragaglai, 2010). O primeiro é a busca por satisfação ligada à funcionalidade dos produtos, na qual, por meio da comparação e preferência por produtos, o consumidor vislumbra as funções para as quais os bens foram originalmente criados (Lindberg, Gärling \& Montgomery, 1989).

O segundo motivo diz respeito à busca por prazer emocional. Esse perfil de consumidor escolhe os produtos de acordo com sua identidade, em busca de autoconhecimento. O consumo, portanto, torna-se mais complexo, pois esses indivíduos não apreciam somente a satisfação das necessidades. Ademais, tais consumidores se distanciam das práticas do consumo por aprovação social, sem levar em consideração os desejos da massa. Essa motivação antagoniza a ideia de que o consumo é "ter", pois os indivíduos dessas classes são caracterizados pela busca de significados, sendo motivados pelo desejo de descobrir quem "são" (Campbell, 2006). Segundo essa vertente do consumo, passamos a conhecer quem somos, a partir do que temos (Muñoz \& Martí, 2008).

Por fim, o terceiro motivo se refere à busca por prazer emocional ligada aos produtos/marca como comunicadores sociais. $O$ desejo de se "encaixar" ou de ser reconhecido em determinado grupo social é o que move as decisões desse tipo de consumidor. Tal motivo tem como principal propósito, segundo Bragaglia (2010, p.109) "a comunicação de status, seja no intuito de 'distinção' visando à 'competição' com alguém, ou de 'inserção' visando o reconhecimento" .

Nessa perspectiva, a modernidade traz um novo hábito de consumo, os indivíduos passam a consumir os símbolos que as mercadorias representam, e não o produto. Esses símbolos são carregados de significados, como o status, que exprimem a posição social que o produto está em relação a outros do mesmo sistema (Slater, 2001). O status, na definição de Strehlau e Aranha (2004, p.10), é "a posição na sociedade atribuída a um indivíduo por outros" - E, segundo Eastman, Goldsmith \& Flynn (1999), ele pode possuir três tipologias: 
1) Status por definição: é atribuído desde a nascença e está relacionado com os valores de aristocracia;

2) Status por realização: é obtido a partir do reconhecimento da superioridade de um indivíduo em relação a outro na realização de uma tarefa;

3) Status por consumo: é obtido através da posse.

O foco deste estudo recai sobre o terceiro tipo de status, definido por Eastman, Goldsmith e Flynn (1999, p.42) como "o processo motivacional pelo qual os indivíduos se esforçam para melhorar sua posição social através do consumo conspícuo de produtos que conferem e simbolizam status para os indivíduos e para os outros".

Corroborando com este posicionamento, O'Cass e Frost (2002, p.68) apresentam o consumo de status como o "processo pelo qual os indivíduos ganham status ou prestígio social a partir da aquisição de bens que os indivíduos e outras pessoas significantes percebem possuir alto status" . Neste tipo de consumo, os indivíduos buscam o reconhecimento de determinada hierarquia social (Strehlau \& Aranha, 2004). Atualizando o conceito para inserir uma visão mais ampla, Eastman e Eastman (2015, p.3) apresentam o consumo de status como "o interesse que o consumidor possui de aumentar sua posição social e/ou self-standing através do consumo de produtos que possam ser conspícuos e que conferem e simbolizam status para os indivíduos e para as pessoas importantes ao seu redor".

Strehlau e Aranha (2004, p. 10) definem produto de luxo como "aquele que provê distinção social por meio de símbolos de status socialmente desejáveis e escassos, preço premium e qualidade". Esses símbolos de status precisam ser socialmente desejados e escassos, quando o produto passa a ser acessível, ele perde o status que possuía (Allérès, 2000). A distinção social e a definição do produto possuir ou não status associado é definido pela percepção das pessoas, cultura e sociedade.

\section{Inovação e inovatividade}


A discussão sobre inovação é bastante corriqueira nos estudos científicos, especialmente por ser algo que está diretamente associada à busca, descoberta, experimentação e à adoção de novos produtos, processos e formas organizacionais (Dosi, Freeman, \& Nelson, 1988). De maneira mais abrangente, seria qualquer novidade percebida pela unidade de adoção, seja uma ideia, prática ou objeto (Rogers, 2003).

O ponto crítico de uma inovação é identificar e reconhecer os impactos que ela causa no comportamento do consumidor (Robertson, 1971). Diante disso, o autor, declara que as inovações podem ser classificadas a partir desses impactos: 1) inovação contínua, aquela que apresenta impacto mínimo sobre o comportamento do consumidor; 2) inovação dinamicamente contínua, aquela que apresenta efeito intermediário sobre o padrão de comportamento do consumidor; e 3) inovação descontínua, aquela que cria um novo padrão de consumo. Semelhante a esta concepção, Tushman e Naldler (1997); Freeman e Perez (1988); Shavinina (2003) distinguem inovação em duas perspectivas: 1) Inovação incremental: referindo-se às inovações básicas, com pequenas modificações, visando o aperfeiçoamento de algo já presente e proporcionando baixa alteração no padrão de consumo; 2) Inovação radical: refere-se às alterações mais expressivas, com novos conceitos, que rompem um senso comum, proporcionando uma quebra no padrão de consumo.

As pessoas aderem às inovações em ritmos diferentes (Rogers, 2003). Diante disso, surge o conceito de inovatividade. As primeiras tentativas de mensurar tal conceito tiveram como instrumento o método do tempo de adoção derivado do conceito estabelecido por Rogers (1971), conforme exposto na Figura 1.

\begin{tabular}{c|l}
\hline Autor & \multicolumn{1}{c}{ Definições } \\
\hline Rogers (1971) & $\begin{array}{l}\text { É o grau em um indivíduo é relativamente precoce na adoção } \\
\text { de inovação, quando comparado com outros membros. }\end{array}$ \\
\hline Hurt, Joseph e Cook & Um dos primeiros estudos de conceitualização, definem \\
\hline
\end{tabular}




\begin{tabular}{c|l}
\hline $\begin{array}{c}\text { (1977) } \\
\begin{array}{c}\text { Midgley e Dowling } \\
\text { (1978) }\end{array}\end{array}$ & $\begin{array}{l}\text { inovação como uma "vontade de mudar". } \\
\text { inata". O grau em que o indivíduo é receptivo a novas ideias e } \\
\text { toma decisões de inovação independentemente da experiência } \\
\text { comunicada de outros. }\end{array}$ \\
\hline Hirschman (1980) & $\begin{array}{l}\text { Concorda parcialmente com a definição anterior no que diz } \\
\text { respeito à influência da inovatividade inata na adoção de } \\
\text { comportamento. "O desejo de procurar o novo e o diferente é } \\
\text { indistinguivel do desejo de adotar novos produtos". Argumenta } \\
\text { que a busca pelo novo e a criatividade trabalham com outras } \\
\text { variáveis para explicar diferentes comportamentos. }\end{array}$ \\
\hline Goldsmith (1990) & Desejo de tentar/experimentar coisas novas. \\
\hline $\begin{array}{c}\text { Engel, Blackwell e } \\
\text { Miniard (2000) }\end{array}$ & $\begin{array}{l}\text { Inovatividade é o grau em que o indivíduo adota uma inovação } \\
\text { relativamente mais cedo do que outros indivíduos. }\end{array}$ \\
\hline
\end{tabular}

Figura 1. Definições de Inovatividade

Nota. Elaborada pelos autores (2019).

Rogers (2003) define cinco tipos ideais de adotantes de inovação baseados no tempo de adoção. Os tipos ideais são conceituações baseadas nas observações projetadas para realizar comparações possíveis. Essas categorias são: Inovadores, Adotantes Iniciais, Maioria Inicial, Maioria Tardia e Retardatários.

- Inovadores (I): são aqueles consumidores obcecados por aventura. Ansiosos por coisas novas e seus interesses os levam do círculo social comum para círculos de relacionamentos mais cosmopolitas.

- $\quad$ Adotantes Iniciais (Al): são mais integrados com o sistema social local. Ao contrário dos inovadores que são cosmopolitas, os Al são locais. É o grupo com o maior grau de liderança de opinião na maior parte dos grupos sociais.

- Maioria Inicial (MI): adotam novas ideias mais rapidamente que a média do seu grupo social. A Ml interage com pessoas próximas, mas raramente está em posição de liderança. 
- Maioria Tardia (MT): adotam novas ideias após a média de adotantes. A adoção pode ser uma necessidade econômica ou uma pressão das relações sociais. Esse grupo adota inovações após a adoção da maioria dos membros de seu grupo.

- Retardatários (R): são os últimos do grupo a adotar uma inovação. São relativamente mais locais e isolados socialmente. Este grupo adota inovação com base no passado e interagem principalmente com pessoas que compartilham os mesmos valores.

Um dos problemas metodológicos que a abordagem de tempo baseada em Rogers (1971) pode provocar é a impossibilidade de avaliar sua confiabilidade e validade. Ainda a generalização só pode ser realizada por indivíduos de um determinado estudo, sendo difícil fazer previsões para comportamento de compra futuro (Goldsmith \& Flynn, 1992).

A tecnologia é um ponto específico dentro da inovação, tornando a mesma, parte integrante desse conceito (Souza, 2002). Diante disso, pesquisadores procuram investigar os determinantes e consequências da adoção de novas tecnologias. Existe um meio para mensuração, o chamado índice de prontidão como "a propensão para adotar e utilizar novas tecnologias seja para atingir objetivos domésticos ou profissionais" (Parasuraman, 2000, p. 308). Segundo o autor, a prontidão a tecnologia pode ser definida em quatro componentes distintos: otimismo, inovatividade, desconforto e insegurança.

Tsikriktsis (2004) explora os quatro componentes apresentados. Otimismo é definido pelo autor como uma visão positiva da tecnologia e uma crença de que ela oferece mais controle, flexibilidade e eficiência em suas vidas. Inovatividade é definida como a tendência que um indivíduo possui de ser um pioneiro na adoção de tecnologia e líder de pensamento. Desconforto é uma percepção de falta de controle ou um sentimento de sobrecarga diante da tecnologia. Por último, o autor define insegurança 
como uma desconfiança em relação à tecnologia e ceticismo em relação à habilidade de funcionar corretamente.

Existem cinco tipos de consumidores de acordo com seus hábitos de consumo de tecnologia (Rita, 2010). são eles: exploradores, pioneiros, céticos, paranóicos e retardatários. Os exploradores são os primeiros a consumir um produto inovador tecnológico quando ele chega no mercado, possuem motivação e falta de medo em relação à compra. Os pioneiros compartilham as crenças dos benefícios da tecnologia, no entanto ainda são inseguros e desconfortáveis no que concerne à adoção. Os céticos precisam ser convencidos dos benefícios da inovação, pois não possuem paixão pela tecnologia. Os paranóicos temem os riscos e exibem grandes graus de insegurança e desconforto, mesmo achando a tecnologia interessante. Já os retardatários, são o grupo oposto aos exploradores, os últimos a adotarem uma nova tecnologia e possuem muitos fatores inibidores. Os componentes de prontidão de tecnologia e suas relações com os consumidores são apresentados abaixo na tabela 2.

\begin{tabular}{c|c|c|c|c}
\hline Segmentos de tecnologia & Otimismo & Inovatividade & Desconforto & Insegurança \\
\hline Exploradores & Alto & Alto & Baixo & Alto \\
\hline Pioneiros & Alto & Alto & Baixo & Baixo \\
\hline Céticos & Baixo & Baixo & Alto & Alto \\
\hline Paranóicos & Alto & Baixo & Baixo & Baixo \\
\hline Retardatários & Baixo & Baixo & Alto & Alto \\
\hline
\end{tabular}

\section{Figura 2: Categorias dos Segmentos de Tecnologia}

Fonte: Demirci e Ersoy (2008).

Em relação ao consumo de produtos inovadores, estes podem ajudar a construir uma imagem positiva, pois o consumidor é capaz de demonstrar suas habilidades perante outros (Costa \& Arruda Filho, 2016). Os consumidores que compõem o perfil de maioria inicial (MI), compram os produtos tecnológicos por serem compatíveis com o que "está na moda", 
para que possam ter os equipamentos atualizados, comprando os produtos com o intuito de apresentá-lo para o grupo e se sentir pertencente (Lucena et al., 2008). Sendo assim, a propensão para a adoção de inovação (inovatividade) pode influenciar essas características sociais. Diante disso, surge a hipótese $\mathrm{Hl}$ :

H1: O grau de inovatividade influencia positivamente a sociabilidade.

Ainda, Lucena et al. (2008) identificaram em sua pesquisa sobre a aquisição de produtos em constante inovação, que uma das principais vantagens para os consumidores inovadores é a compra para obtenção de prestígio social. Eastman e Eastman (2015) explanam que os produtos podem ser comprados pelo seu valor simbólico e social ao invés de sua utilidade funcional. Portanto, os produtos tecnológicos podem possuir status atrelado, indicando que os consumidores, com propensão para adotar uma inovação mais cedo, podem ter o consumo por status como motivação, ultrapassando a compra somente pela natureza funcional dos produtos (nãofuncionalidade). Diante disso, apresentam-se as hipóteses H2 e H3:

H2: O grau de inovatividade influencia positivamente o consumo de status.

H3: O grau de inovatividade influencia positivamente a nãofuncionalidade.

Propondo uma escala curta, válida e confiável, Goldsmith e Hofacker (1991) apresentam a Escala de Domínio-Específico de Inovatividade, composta por seis itens e validada a partir de uma pesquisa com 135 mulheres. A escala foi novamente testada por Goldsmith (2001) com o propósito de explorar sua utilidade para fins de revalidação, para compreender e prever o comportamento do consumidor da internet. A confiabilidade foi comprovada e o autor sugere que outros pesquisadores proponham e testem hipóteses relacionando a inovatividade com outros construtos. 


\section{MÉTODO}

Nesta seção são expostos os procedimentos metodológicos adotados para realização da pesquisa de campo. Utilizamos uma abordagem quantitativa com coleta de dados primários por meio de survey com jovens universitários.

Para a coleta de dados, utilizou-se como instrumento de pesquisa o questionário composto por questões sociodemográficas (sexo, idade, estado civil, escolaridade, renda familiar do respondente); pela Escala de DomínioEspecífico de Inovatividade (seis itens) elabora por Goldsmith (1990); e pela Escala de Consumo de Status criada por Eastman, Goldsmith e Flynn (1999), traduzida e adaptada ao Brasil por Strehlau e Aranha (2004). Esta última está dividida em três dimensões: Sociabilidade (cinco itens), Status (cinco itens) e Não-funcionalidade (quatro itens). Todas as variáveis foram mensuradas por meio de escala tipo Likert de sete pontos, variando de 1 (Discordo totalmente) a 7 (Concordo totalmente).

Para a escala de inovatividade de Goldsmith (1990), foi efetuada a tradução e adaptação dos itens para a contextualização no âmbito de produtos tecnológicos, seguindo a orientação dos autores da escala, a qual deveria ser aplicada considerando os objetivos de cada pesquisa. Já a escala de Consumo de Status, apenas a adaptação para o contexto de produtos tecnológicos foi procedida, inicialmente.

Finalizada a adaptação dos itens, foi realizada a estruturação do questionário de modo que os itens de cada construto ficassem misturados para evitar viés de respostas dos inquiridos. Terminada esta etapa, foi feito a aplicação do pré-teste com 17 respondentes, estudantes universitários, com o intuito de verificar eventuais erros e não compreensão dos itens. Posteriormente, foram necessárias pequenas adaptações em alguns itens da escala validada ao Brasil. Por exemplo, no item "Eu comprarei um novo produto tecnológico, mesmo se eu ainda não tiver ouvido falar sobre ele" ficou "Eu compraria um novo produto tecnológico, mesmo se eu ainda não 
tiver ouvido falar sobre ele". Finalizados os ajustes, procedemos a coleta dos dados, que foi feita por conveniência e acessibilidade dos pesquisadores.

A etapa de coleta de dados ocorreu entre os meses de março a junho de 2018 e de duas formas: online e presencial. Para a aplicação online, o questionário foi estruturado na plataforma Google Forms e o link do instrumento foi compartilhado nas redes sociais, Facebook, Instagram e WhatsApp, dos autores e de amigos. A primeira parte do questionário online informava o objetivo da pesquisa e se o respondente concordava em participar voluntariamente do estudo; e as demais continham os itens da escala e questões sociodemográficas.

A aplicação presencial ocorreu em instituições de ensino médio e superior, tanto públicas quanto privadas, em três cidades do Nordeste brasileiro e foi realizada pelos pesquisadores. Antes de responder ao questionário, os participantes assinaram Termo de Consentimento Livre e Esclarecido (TCLE) informando sobre os objetivos e sobre a participação voluntária no estudo. Posteriormente, o questionário era entregue ao inquirido. Finalizada a etapa de coleta dos dados, realizou-se a tabulação dos dados aplicados de maneira presencial junto aos dados online no SPSS (Statistical Package for Social Sciences) versão 22.

Para as análises dos dados, inicialmente foi realizada uma análise exploratória com o intuito de verificar missing values e outliers. No total obtivemos 826 respostas e, após procedimentos de limpeza, quatro foram deletadas por possuírem missing values superiores a 5\% do questionário. Neste sentido, ficaram 822 entradas válidas para as análises posteriores.

As duas escalas utilizadas possuíam itens reversos, de modo que foi procedida a inversão da pontuação para não comprometer as análises seguintes. Como forma de verificar a adequação dos construtos, procedeuse à extração da consistência interna, por meio do Alfa de Cronbach, além de análises descritivas das dimensões. 
Para a análise das hipóteses e para outras verificações, uma variedade de técnicas foi utilizada. Inicialmente efetuamos a regressão linear e depois realizamos o teste de associação por meio da correlação de Pearson para avaliação dos construtos dois a dois. Em complemento, também executamos testes para verificar possíveis diferenças nos construtos considerando algumas variáveis sociodemográficas. Todos os procedimentos foram operacionalizados no SPSS e baseados na literatura especializada (Field, 2009; Hair et al., 2009; Costa, 2011).

\section{RESULTADOS}

Nesta seção são expostos os resultados da pesquisa e os procedimentos estatísticos. Ordenadamente, são apresentados a descrição da amostra, seguida da análise psicométrica dos construtos, do teste das hipóteses e de análises complementares entre os construtos e algumas variáveis sociodemográficas.

\section{Descrição da amostra}

A pesquisa foi realizada com 822 estudantes do ensino médio e jovens universitários, onde 52,45\% são estudantes da rede pública ( $N=431$ ), 46,7\% da rede privada $(\mathrm{N}=384), 0,85 \%(\mathrm{~N}=7)$ não informaram. Sobre o nível de escolaridade $73,8 \%$ são estudantes do ensino superior $(N=607)$ e 26,2\% são estudantes do ensino médio $(n=215)$. Dessa amostra 69\% são questionários físicos ( $N=567)$ e $31 \%$ foram aplicados online $(N=255)$. Entre os entrevistados $58,15 \%$ são do sexo feminino ( $N=478), 41,73 \%$ do sexo masculino $(N=343)$ e $0,12 \%$ não se identificaram $(N=1)$.

Dentre os entrevistados, o respondente de menor idade tinha 14 anos, o de maior idade 30 anos e a média de idade foi 20 anos. A mediana referente à idade também foi 20 anos e moda de 18 anos. Por se tratar de um público jovem 44,77\% não possuem renda individual ( $N=368), 14,6 \%$ recebem mesada ( $N=120), 15,8 \%$ trabalham $(N=130), 6,2 \%$ são estagiários $(\mathrm{N}=51), 4,15 \%$ são autônomos ( $\mathrm{N}=34), 10,1 \%$ são bolsistas universitários ( $N=83)$, 
1,95\% recebem auxílio universitário ( $N=16), 1,2 \%$ possuem outro tipo de renda individual $(\mathrm{N}=10)$ e 1,2\% não responderam.

Em relação a renda mensal familiar, 7,55\% possuem renda inferior a um salário mínimo (N=62), 34,1\% possuem renda de 1 a 2 salários mínimos $(N=280), 20,3 \%$ possuem renda de 2 a 3 salários mínimos ( $N=167), 22,5 \%$ possuem renda de 3 a 6 salários mínimos ( $N=185)$, 10,45\% somam de 6 a 12 salários mínimos ( $\mathrm{N}=86), 3,4 \%$ possuem de 12 a 26 salários mínimos ( $\mathrm{N}=28)$ e apenas $1,7 \%$ possuem acima de 26 salários mínimos ( $N=14)$.

O estado civil predominante foi o de solteiro com 94,1\% ( $N=773$ ), este resultado se justifica devido a amostra ser composta exclusivamente por jovens. Logo após estão os casado/ união estável com 5,35\% ( $N=44)$, em seguida, os separado/divorciado com 0,36\% (N=3) e por último os viúvo com $0,12 \%(N=1)$.

Referente à localização dos entrevistados, 0,72\% (N=6) não responderam. O estado com maior número de respondente foi o Ceará - CE com 87,46\% ( $N=719)$, em seguida vem os entrevistados da Paraíba - PB com 4,13\% ( $N=34)$, Bahia - $B A$ com 3,9\% ( $N=32)$, Pernambuco - PE com 1,9\% ( $N=16)$ e $1,85 \%$ são de outros estados $(N=15)$.

\section{Análise psicométrica dos construtos}

Com o intuito de verificar a adequação dos construtos para as análises de confiabilidade, de descrição e das hipóteses. A princípio, a primeira extração foi a medida de confiabilidade por meio do alpha de Cronbach (mínimo de 0,600).

Quanto aos resultados encontrados nas afirmativas da escala, no construto de Inovatividade, composto por 6 itens, a média de resposta do construto foi de 3,40 e a mediana de 3,33, o que indica que os participantes da pesquisa possuem pouco comportamento inovador quando se trata de produtos tecnológicos. O desvio padrão se manteve num nível baixo, indicando pouca variabilidade nos dados. O alpha de Cronbach sinaliza um 
valor baixo (0,609), mas ainda assim acima do mínimo aceitável (Costa, 2011 ). Verificando os itens, é possível notar que as variáveis relacionadas ao aspecto tecnológico no círculo de amigos (INOV1, INOV3 e INOV5) apresentaram as maiores medidas, com destaque para a média e mediana do item INOV5, com valores de 4,67 e 5,0, respectivamente, conforme detalhado na Tabela 1.

Tabela 1:

Medidas psicométricas da escala de Inovatividade

\begin{tabular}{|c|c|c|c|c|}
\hline & Alfa & Média & Mediana & $\begin{array}{l}\text { Desvio } \\
\text { padrão }\end{array}$ \\
\hline & 0,609 & 3,40 & 3,33 & 1,05 \\
\hline \multicolumn{2}{|r|}{ Itens de Inovatividade } & Média & Mediana & $\begin{array}{l}\text { Desvio } \\
\text { padrã } \\
0\end{array}$ \\
\hline INOVI* & $\begin{array}{l}\text { Em geral, eu sou a (o) última (o) do meu } \\
\text { círculo de amizades a comprar produtos } \\
\text { tecnológicos quando o item surge. }\end{array}$ & 3,47 & 3,0 & 1,87 \\
\hline INOV2 & $\begin{array}{l}\text { Se eu ouço/escuto que um produto } \\
\text { tecnológico novo está disponível na loja, eu } \\
\text { fico bastante interessado (a) em comprá-los. }\end{array}$ & 3,41 & 3,0 & 1,87 \\
\hline INOV3* & $\begin{array}{l}\text { Comparado (a) aos meus amigos(as) eu } \\
\text { possuo poucos itens de produtos } \\
\text { tecnológicos. }\end{array}$ & 3,82 & 4,0 & 1,82 \\
\hline INOV4 & $\begin{array}{l}\text { Eu comprarei um novo item tecnológico, } \\
\text { mesmo se eu ainda não tiver ouvido falar } \\
\text { sobre ele. }\end{array}$ & 2,05 & 2,0 & 1,46 \\
\hline INOV5* & $\begin{array}{l}\text { Em geral, eu sou o(a) último(a) do meu } \\
\text { círculo de amigos a saber o nome dos } \\
\text { produtos tecnológicos mais recentes. }\end{array}$ & 4,67 & 5,0 & 1,93 \\
\hline INOV6 & $\begin{array}{l}\text { Eu conheço o nome dos produtos } \\
\text { tecnológicos antes das outras pessoas. }\end{array}$ & 2,98 & 2,0 & 1,82 \\
\hline
\end{tabular}

Nota. * Item com pontuação revertida para análise. 
Na dimensão Status, composto por 5 itens, a média dos respondentes foi de 2,45. Este resultado indica que no construto analisado, os participantes discordam que compram produtos inovadores para obtenção de status, fato acompanhado pela mediana que demonstra que metade dos respondentes pontuaram até 2,20 na escala, que variava de 1 a 7 . O alpha de Cronbach obtido foi de 0,730, um número satisfatório e que indica a boa confiabilidade do construto. Os dados estão na Tabela 2, a seguir.

Tabela 2:

Medidas psicométricas da escala de Status

\begin{tabular}{cccc} 
Alfa & Média & Mediana & $\begin{array}{c}\text { Desvio } \\
\text { padrão }\end{array}$ \\
\hline 0,730 & 2,45 & 2,20 & 1,13 \\
\hline & Média & Mediana & $\begin{array}{c}\text { Desvio } \\
\text { padrão }\end{array}$
\end{tabular}

\begin{tabular}{llcccc}
\hline \multicolumn{1}{c}{ Itens de Status } & Média & Mediana & $\begin{array}{c}\text { Desvio } \\
\text { padrão }\end{array}$ \\
\hline STA1 & $\begin{array}{l}\text { Eu compraria um produto somente porque ele } \\
\text { me dá status. }\end{array}$ & 1,91 & 1,0 & 1,41 \\
\hline STA2 & $\begin{array}{l}\text { Eu me interesso por novos produtos que dão } \\
\text { status. }\end{array}$ & 2,16 & 2,0 & 1,52 \\
\hline STA3 & Eu pagaria mais por produtos de mais status. & 2,85 & 2,0 & 1,74 \\
\hline STA4* & O status que um produto me dá é irrelevante. & 3,10 & 2,0 & 1,93 \\
\hline STA5 & Um produto é mais valioso pra mim se tiver grife. & 2,25 & 2,0 & 1,55 \\
\hline
\end{tabular}

Nota. * Item com pontuação revertida para análise.

Conforme exposto na Tabela 2, no construto sociabilidade, composto por 5 itens, a média de respostas obtidas foi de 5,50, este resultado transmite que a amostra analisada possui um alto índice de sociabilidade e são opostos ao comportamento antissocial. Esse comportamento se deve ao fato de todos os itens apresentarem médias acima de 5,00, com exceção de soc3. O alpha de Cronbach obtido com o construto foi de 0,720 , indicando boa confiabilidade do construto.

Tabela 3:

Marketing \& Tourism Review • Belo Horizonte - MG - Brasil • v. 5, n. 1, mai - jun, 2020

NEECIM TUR • Núcleo de Estudos e Estratégias em Comunicação Integrada de Marketing e Turismo • UFMG 


\begin{tabular}{|c|c|c|c|c|c|}
\hline & & Alfa & Média & Mediana & $\begin{array}{l}\text { Desvio } \\
\text { padrão }\end{array}$ \\
\hline & & 0,720 & 5,50 & 5,80 & 1,15 \\
\hline \multicolumn{3}{|c|}{ Itens de Sociabilidade } & Média & Mediana & $\begin{array}{l}\text { Desvio } \\
\text { padrão }\end{array}$ \\
\hline $\mathrm{SOCl}^{*}$ & \multicolumn{2}{|l|}{ Eu não me considero uma pessoa sociável. } & 5,138 & 6,0 & 1,89 \\
\hline SOC2* & \multicolumn{2}{|l|}{ Eu não aprecio eventos sociais. } & 5,542 & 6,0 & 1,72 \\
\hline SOC3* & \multicolumn{2}{|c|}{ Eu prefiro estar só do que rodeado de pessoas. } & 4,985 & 5,0 & 1,77 \\
\hline SOC4 & \multicolumn{2}{|l|}{ Eu aprecio sair com grupos de pessoas. } & 5,99 & 6,0 & 1,40 \\
\hline SOC5* & \multicolumn{2}{|l|}{ Eu não gosto de conhecer pessoas novas. } & 5,842 & 6,0 & 1,55 \\
\hline
\end{tabular}

Nota. * Item com pontuação revertida para análise.

Nas análises do construto Não funcionalidade, composto inicialmente por 4 itens, tivemos que excluir a variável FUN2 com o intuito de melhorar o alpha para 0,521. No entanto, ainda assim, ele se manteve abaixo do aceitável que seria pelo menos de 0,600 (Costa, 2011). O construto apresentou uma média de 3,21, indicando que os consumidores que compram produtos tecnológicos buscam nesses produtos seus aspectos funcionais. No entanto, o desvio padrão do construto ficou em um nível alto, indicando variabilidade dos dados, conforme se observa na Tabela 4.

Tabela 4:

Medidas psicométricas da escala de Não Funcionalidade

\begin{tabular}{llcccc}
\hline & Alfa & Média & Mediana & $\begin{array}{c}\text { Desvio } \\
\text { padrão }\end{array}$ \\
\cline { 3 - 5 } & 0,521 & 3,21 & 3,00 & 1,84 \\
\hline Itens para Não Funcionalidade & Média & Mediana & $\begin{array}{c}\text { Desvio } \\
\text { padrão }\end{array}$ \\
\hline FUN1* & $\begin{array}{l}\text { É bobagem comprar produtos que não } \\
\text { sejam práticos. }\end{array}$ & 3,22 & 3,0 & 1,48 \\
\end{tabular}




\begin{tabular}{llllll}
\hline FUN2* & $\begin{array}{l}\text { Eu não gastaria dinheiro com produtos } \\
\text { práticos. }\end{array}$ & - & - \\
\hline FUN3 & $\begin{array}{l}\text { Eu compro produtos por razões não } \\
\text { funcionais. }\end{array}$ & 2,34 & 2,0 & 1,54 \\
\hline FUN4* & $\begin{array}{l}\text { Eu só compro produtos que tenham um } \\
\text { propósito funcional. }\end{array}$ & 2,44 & 2,0 & 1,85 \\
\end{tabular}

Nota. ${ }^{*}$ Item com pontuação revertida para análise.

Finalizada a etapa psicométrica dos construtos e de sua adequação, as análises dos dados continuaram. No próximo tópico são expostos os resultados dos testes de hipóteses por meio da análise de regressão e por teste de associação por meio da correlação de Pearson.

\section{Testes de hipóteses}

Como forma de testar as hipóteses da presente pesquisa, foi executado a regressão linear. Inicialmente verificamos o coeficiente padronizado Beta de influência linear entre os construtos.

Com base nos dados da Tabela 5, é possível verificar que as hipóteses de que a Inovatividade influencia positivamente na Sociabilidade $(\beta=0,127$, $p<0,001)$; no Status $(\beta=0,336, p<0,001)$ e na Não funcionalidade $(\beta=0,313$, $p<0,001)$ foram aceitas. Considerando os valores de beta ( $\beta$ ), percebemos que a inovatividade apresenta maiores poderes de previsão para o Status e para a Não funcionalidade no contexto aqui abordado. O status, portanto, é o principal fator quando consideramos o consumo de produtos tecnológicos (Nascimento, Oliveira, \& Cenerino, 2013).

Tabela 5:

Teste de Hipótese

\begin{tabular}{cccccc}
\hline Hipótese & Relação & $\boldsymbol{B}$ & p-valor & $\mathbf{R}^{2}$ & Resultado \\
\hline $\mathrm{H} 1(+)$ & Inovatividade-> Sociabilidade & 0,127 & 0,000 & 0,015 & Confirmada \\
\hline $\mathrm{H} 2(+)$ & Inovatividade -> Status & 0,336 & 0,000 & 0,112 & Confirmada \\
\hline
\end{tabular}


Ademais, ainda considerando os dados da Tabela 5, percebe-se que na amostra investigada os objetos tecnológicos são utilizados como marcadores de status. No entanto, no processo de sociabilidade, apesar de significativa, tal influência se dá de maneira tímida. Tais observações são reforçadas ao verificar o coeficiente de determinação $\left(R^{2}\right)$, os quais ficaram em níveis muito baixos. O melhor desempenho de explicação da variação, considerando a Inovatividade, foi a relação com o Status com $R^{2}=0,112$.

Como forma de melhor verificar as relações das hipóteses, efetuamos o teste de associação por meio da correlação de Pearson. Considerando as correlações entre os construtos das hipóteses da pesquisa, nota-se que todas as relações se mantém positivas e significativas com valores de 0,127;0,313 e 0,336 para Sociabilidade, Não Funcionalidade e Status, respectivamente. Todas as correlações estão expostas na Tabela 6.

Tabela 6:

Correlação de Pearson entre os construtos

\begin{tabular}{ccccc}
\hline & Inovatividade & Sociabilidade & Status & $\begin{array}{c}\text { Não } \\
\text { Funcionalidade }\end{array}$ \\
\hline Inovatividade & 1,000 & $0,127^{* *}$ & $0,336^{* *}$ & $0,313^{* *}$ \\
\hline Sociabilidade & 1,000 & 0,031 & $0,139^{* *}$ \\
\hline Status & & 1,000 & $0,296^{* *}$ \\
\hline Não Funcionalidade & & & 1,000 \\
\hline
\end{tabular}

Nota. ${ }^{* *}$ A correlação é significativa no nível 0,01.

De modo geral, as correlações corroboram os resultados da regressão executada anteriormente, ou seja, as variações do consumo por status de objetos tecnológicos é pouco explicado a partir do comportamento inovativo. Dessa forma, algumas análises estatísticas complementares são 
apresentadas, a fim de ampliar a compreensão do fenômeno de compra de objetos tecnológicos inovadores e sua relação com o consumo por status.

\section{Análises complementares}

Com o intuito de analisar as relações dos construtos Inovatividade e Consumo de status com algumas variáveis sociodemográficas, foram realizadas extrações complementares considerando o sexo e o tipo de ensino. Para essas análises complementares, consideramos a escala de Consumo de Status, formada pela média aritmética das três dimensões (Sociabilidade, Status e Não-funcionalidade).

Devido ao tamanho da amostra, as medidas de posição (média, mediana e moda), do histograma das variáveis inovatividade e consumo de status, verificou-se que os dados se aproximam de uma distribuição normal, de modo que se procedeu à realização de testes paramétricos.

\section{Análise por sexo}

A primeira análise, feita por meio do Teste $t$, complementar realizada considerou o sexo dos indivíduos. Para ambos os casos (inovatividade e consumo de status), o teste de Levene foi não significativo, sinalizando que a suposição de homogeneidade está satisfeita (Field, 2009). Conforme observamos na Tabela 7, os resultados mostram que, para a variável Inovatividade, as diferenças de médias entre os sexos se confirmam $t(816)=$ $6,400 p<0,05$.

Tabela 7:

Comparação por gênero

\begin{tabular}{ccccc|c}
\hline & F & Sig & $\boldsymbol{f}$ & $\begin{array}{c}\text { Graus de } \\
\text { liberdade }\end{array}$ & Sig \\
\hline Inovatividade & 1,095 & 0,296 & $-6,400$ & 816 & 0,000 \\
\hline Consumo de status & 1,678 & 0,196 & $-0,932$ & 807 & 0,352 \\
\hline
\end{tabular}

Fonte: dados da pesquisa (2018). 
Considerando os resultados apresentados na Tabela 7 para a variável Consumo de Status, percebe-se que as médias por gênero não são estatisticamente diferentes $(t(807)=-0,932 p>0,05)$. Assim, homens e mulheres apresentam comportamentos semelhantes quando se considera o consumo por status.

\section{Tipo de ensino}

Ainda como forma de melhor averiguar possíveis divergências em relação a algumas variáveis sociodemográficas, efetuamos análises considerando o tipo de ensino da amostra (se público ou privado). Na Tabela 8, a seguir, podemos verificar que o construto Inovatividade apresentou resultados significativos $(t(814)=-3,099 p<0,05)$, mostrando que existem diferenças em média para inovatividade pelo tipo de ensino da amostra. Pessoas do ensino público obtiveram média maior $(3,52)$ que as pessoas do ensino público $(3,29)$.

Tabela 8:

Comparação por tipo de ensino

\begin{tabular}{ccccc|c}
\hline & F & Sig & $\boldsymbol{\dagger}$ & $\begin{array}{c}\text { Graus de } \\
\text { liberdade }\end{array}$ & Sig \\
\hline Inovatividade & 0,490 & 0,484 & $-3,099$ & 814 & 0,002 \\
\hline Consumo de status & 0,352 & 0,553 & $-0,252$ & 805 & 0,801 \\
\hline
\end{tabular}

Ainda de acordo com os resultados da tabela 8, o construto Consumo de Status não obteve resultados significativos $(t(805)=-0,252 p>0,05)$. Ao analisar as médias, percebemos que pessoas do ensino público tem média de 3,77, enquanto que as do ensino privado apresentam média de 3,79.

\section{Discussão dos resultados}

Os resultados obtidos pelos testes de hipóteses indicam que a inovatividade possui relação positiva com a sociabilidade, confirmando 
assim a $\mathrm{Hl}$ (O grau de inovatividade influencia positivamente a sociabilidade). De acordo com Eastman, Goldsmith e Flynn (1999), entendese que os consumidores de status buscam produtos que são reconhecidos pelos outros como possuidores de status. Essa ideia afirma a importância dos grupos de referências no processo de compra dos produtos tecnológicos. Lucena et al., (2008) discorrem que os indivíduos classificados como inovadores e adotantes iniciais são vistos como "grupo de referência" pelos demais consumidores, dado os níveis de conhecimento técnicos em relação a tecnologia e pela capacidade de justificar a aquisição desses determinados produtos. Portanto, uma vez que um indivíduo se sente motivado ou enxerga a oportunidade de melhorar a sua posição social a partir da compra de produtos tecnológicos, ele ratifica a relação existente entre o consumo de produtos tecnológicos inovadores com a sociabilidade.

Diante dos achados, é possível identificar que os produtos tecnológicos inovadores possuem o status como um dos motivos que leva o consumidor a comprar tais bens. Ao consumir por status, os indivíduos procuram reconhecimento em certa hierarquia social (Strehlau \& Aranha, 2004). A relação forte encontrada entre o status e a inovatividade nos indica que os produtos tecnológicos inovadores são considerados para os consumidores como produtos que possuem status atrelados e que podem promover reconhecimento social, confirmando a $\mathrm{H} 2$ (O grau de inovatividade influencia positivamente o consumo de status). Dessa forma, alguns consumidores veem o consumo de produtos tecnológicos como forma de adquirir status, a fim de pertencer ou se diferenciar de determinado grupo. Este resultado corrobora com as teorias de Simmel (1904) e Bourdieu (2001). Sendo assim, é possível identificar que os produtos eletrônicos investigados são percebidos pelos indivíduos como possuidores de status, reforçando a discussão de Bilge (2015) de que o bem de luxo depende da percepção dos indivíduos, da cultura e da sociedade.

Ademais, o teste de hipóteses também nos permite compreender que os produtos tecnológicos podem ser comprados por razões que vão além da 
sua funcionalidade, confirmando a H3 $(\mathrm{O}$ grau de inovatividade influencia positivamente a não-funcionalidade). No geral, produtos podem ser comprados pelo valor social e/ou simbólico e também podem ser usados para demonstrar riqueza, sucesso, exclusividade ou identidade pessoal (Eastman \& Eastmn, 2015). Os resultados indicam que os consumidores de produtos tecnológicos inovadores investigados, buscam, ao consumir tais bens, benefícios que transcendem a funcionalidade destes. Além disso, os resultados indicam a ideia apresentada por Dogan et al., (2018) de que os produtos luxuosos são comprados como forma de construção e de presentear o self. Diante disso, os consumidores procuram nos produtos tecnológicos uma forma de apresentar sua identidade e de buscar identificação com o grupo em que faz parte ou que deseja ser parte.

\section{CONSIDERAÇÕES FINAIS}

Este estudo teve como objetivo analisar a relação entre o grau de adoção de produtos tecnológicos inovadores e o consumo de status, identificando como o consumo de status pode ser influenciado pela propensão do indivíduo de adotar uma inovação mais cedo que outros do seu grupo social. Tal estudo foi realizado com base na escala de Inovatividade de Goldsmith e Hofacker (1990) e nos três construtos que compõem a escala de consumo de status (SCS) desenvolvida por Eastman, Goldsmith e Flynn (1999): sociabilidade, status e não-funcionalidade. As duas escalas foram utilizadas para verificar a relação entre a Inovatividade e o consumo de status.

Por meio da literatura e deste estudo de cunho quantitativo realizado, nas análises dos dados foi verificado que o grau de adoção de inovação influencia o consumo de Status, Sociabilidade e a Não-funcionalidade no contexto de produtos tecnológicos inovadores. No entanto, tal influência, apesar de significativa, mantém-se em um nível tímido, indicando que outras variáveis podem melhor explicar a variação nos construtos dependentes. 
A $\mathrm{Hl}(\mathrm{O}$ grau de inovatividade influencia positivamente a sociabilidade) foi confirmada, apesar de maneira fraca $\left(R^{2}=0,015, \beta=0,127\right.$, $p<0,001)$, indicando que o grau de adoção de produtos tecnológicos tem uma implicação direta com a sociabilidade, com os indivíduos buscando, além de prestígio, o estabelecimento de relações social.

A H2 (O grau de inovatividade influencia positivamente o consumo de status) apresenta resultados que indicam uma relação positiva entre a Inovatividade e o Consumo de Status $\left(R^{2}=0,112, \beta=0,336, p<0,001\right)$, este resultado implica a Inovatividade como um construto relevante no consumo de Status, indicando que o grau de adoção de produtos tecnológicos impacta no consumo por status destes bens. Este resultado aponta que os consumidores destes produtos enxergam em tais objetos, significados simbólicos, entre eles e o status.

A terceira e última hipótese (O grau de inovatividade influencia positivamente a não-funcionalidade) também foi confirmada $\left(R^{2}=0,097\right.$, $\beta=0,313, \quad p<0,001)$ apontando que os consumidores de produtos tecnológicos, buscam os bens por motivos que ultrapassam o funcional, entre esses motivos, o próprio status, confirmado pela hipótese anterior. Futuras pesquisas podem ser realizadas para compreender outras variáveis que impactam na decisão de compra desses consumidores, tendo em vista que apesar das relações positivas os coeficientes de explicação para cada hipótese ficaram em níveis baixos.

Em um campo teórico, este artigo apresenta contribuições para os estudos de marketing que abordam consumo de status por jovens, especialmente aos produtos tecnológicos. Os resultados aqui encontrados podem disparar discussões a respeito do uso de tecnologias no processo de socialização do consumidor e de seu uso como marcador de status social, especialmente por jovens. Em um aspecto de mercado, os achados contribuem para melhor compreender o uso de objetos tecnológicos pelo 
grupo abordado e de dar possíveis direcionamentos em campanhas publicitárias voltadas a este público.

Em relação às limitações, primeiramente mencionamos a amostra, que apesar de significativa para boa parte das pesquisas em Administração, ficou restrita, em sua maioria, à região Nordeste do Brasil. Neste sentido, recomenda-se que a pesquisa seja replicada em outros contextos e em outras regiões para averiguar possíveis semelhanças ou divergências de resultados. Outra limitação da pesquisa reside nos itens da escala de Consumo de Status, dividida em três dimensões pois, boa parte das afirmações são compostas por itens reversos, de modo que se recomenda que novos estudos possam ser desenvolvidos com itens não reversos.

Além disso, foram analisados poucos construtos. Outras pesquisas, que estudem a relação entre o status e a inovatividade podem ser realizadas para uma visão mais abrangente do fenômeno. Abordagens, como a quantitativa, podem ser utilizadas para investigar o fenômeno do consumo de status com mais profundidade.

\section{REFERÊNCIAS}

Allérès, D. (2000). Luxo... estratégias marketing. FGV Editora.

Barboza, M. N. L., \& Arruda Filho, E. J. M. (2012). O comportamento do consumidor tecnológico diante dos valores ecologicamente corretos: ideologia verde versus responsabilidade social. Intercom-Revista Brasileira de Ciências da Comunicação, 35(1).

Bilge, S. (2013). Intersectionality undone: Saving intersectionality from feminist intersectionality studies. Du Bois Review: Social Science Research on Race, $10(2), 405-424$.

Belk, R. W. (2013). Extended self in a digital world. Journal of Consumer Research, 40(3), 477-500. 
Bourdieu, P. (2003). Las estructuras sociales de la economía.

Bragaglia, A. P. (2010). Comportamentos de consumo na contemporaneidade. Comunicação Mídia e Consumo, 7(19), 107-124.

Campbell, C. (2006). Eu compro, logo sei que existo: as bases metafísicas do consumo moderno. Cultura, consumo e identidade. Rio de Janeiro: FGV, 4764.

Christo, N., \& Demuner, J. A. (2017) Fatores de Motivação do Consumo por Status de Bens de Luxo por Jovens Brasileiros. In: Seminário em Administração.

Cortez, A. T. C. (2009). Consumo e desperdício: as duas faces das desigualdades. São Paulo: UNESP.

Costa, E. M. S., \& Arruda Filho, E. J. M. (2016, September). Prestígio como Motivador das Intenções de Consumo Tecnológico: Uma Revisão Conceitual. In CLAV 2016.

Costa, F. D. (2011). Mensuração e desenvolvimento de escalas: aplicações em administração. Rio de Janeiro: Ciência Moderna.

Demirci, A. E., \& Ersoy, N. F. (2008). Technology readiness for innovative hightech products: how consumers perceive and adopt new technologies. The Business Review, 11 (1), 302-308.

Dosi, G., Freeman, C., Nelson, R., Silverberg, G., \& Soete, L. (1988). Technical change and economic theory. Laboratory of Economics and Management (LEM), Sant'Anna School of Advanced Studies, Pisa, Italy.

Eastman, J. K., \& Eastman, K. L. (2015). Conceptualizing a model of status consumption theory: An exploration of the antecedents and consequences of the motivation to consume for status. Marketing Management Journal, $25(1), 1-15$

Eastman, J. K., Goldsmith, R. E., \& Flynn, L. R. (1999). Status consumption in consumer behavior: Scale development and validation. Journal of marketing theory and practice, 7(3), 41-52. 
Engel, B., \& Blackwell, R. (1988). Consumer Behavior. Marketing research. Harcourt Australia. International, 8.

Field, A. (2009). Descobrindo a estatística usando o SPSS-2. Bookman Editora.

Freeman, C., \& Perez, C. (1988). Structural crises of adjustment: business cycles. Technical change and economic theory. Londres: Pinter.

Dosi, G., Freeman, C., Nelson, R., Silverberg, G., \& Soete, L. (1988). Technical change and economic theory. Laboratory of Economics and Management (LEM), Sant'Anna School of Advanced Studies, Pisa, Italy.

Goldsmith, R. E. (1991). The validity of a scale to measure global innovativeness. Journal of Applied Business Research, 7(2), 89-97.

Goldsmith, R. E. (2001). Using the domain specific innovativeness scale to identify innovative internet consumers. Internet Research, 11 (2), 149-158.

Goldsmith, R., \& Reinecke Flynn, L. (1992). Identifying innovators in consumer product markets. European Journal of Marketing, 26(12), 42-55.

Goldsmith, R. E., \& Hofacker, C. F. (1991). Measuring consumer innovativeness. Journal of the academy of marketing science, 19(3), 209-221.

Hair, J. F., Black, W. C., Babin, B. J., Anderson, R. E., \& Tatham, R. L. (2009). Análise multivariada de dados. Bookman Editora.

Hirschman, E. C. (1980). Innovativeness, novelty seeking, and consumer creativity. Journal of consumer research, 7(3), 283-295.

Hurt, H. T., Joseph, K., \& Cook, C. D. (1977). Scales for the measurement of innovativeness. Human Communication Research, 4(1), 58-65.

Kerviler, G., \& Rodriguez, C. M. (2019). Luxury brand experiences and relationship quality for Millennials: The role of self-expansion. Journal of Business Research. 
Lindberg, E., Gärling, T., \& Montgomery, H. (1989). Differential predictability of preferences and choices. Journal of Behavioral Decision Making, 2(4), 205219.

Midgley, D. F., \& Dowling, G. R. (1978). Innovativeness: The concept and its measurement. Journal of consumer research, 4(4), 229-242.

Martí, J., Muñoz, P., \& Medina, A. (2008). Engagement marketing: una nueva publicidad para un marketing de compromiso. Prentice Hall/Financial Times.

Nascimento, M. R., de Oliveira, J. S., \& Cenerino, A. (2014). A influência da imersão social na constituição dos significados do consumo e na adoção de produtos tecnológicos por adolescentes: uma análise teórica a partir da sociologia econômica. Perspectivas Contemporâneas, 8(2).

O'cass, A., \& Frost, H. (2002). Status brands: examining the effects of nonproduct-related brand associations on status and conspicuous consumption. Journal of product \& brand management, $11(2), 67-88$.

Parasuraman, A. (2000). Technology Readiness Index (TRI) a multiple-item scale to measure readiness to embrace new technologies. Journal of service research, 2(4), 307-320.

Peixoto, A. F., Carneiro, J. S., Baldanza, R. F., LEAL, J. S., \& Pellizzoni, L. N. (2018). Tecnologias móveis e consumo por adolescentes: um olhar sobre a percepção dos pais e filhos. In: Encontro de Marketing da Anpad, 2018.

Pereira, N. A. C., \& Teixeira, A. (2018). Fatores de Motivação no Consumo de Itens de Luxo. Revista Brasileira de Marketing, 17(3), 401-414.

Rita, L. P. S., de Araújo, A. C., de Paula, M. A., de Oliveira Lima, M., \& Viana Filho, J. C. (2010). Consumo de produtos e serviços inovadores: aplicação do índice de prontidão para tecnologia. Revista Brasileira de Inovação, 9(1), 167-196.

Robertson, T. S. (1971). Innovative behavior and communication. Holt McDougal. 
Rogers, E. M. (2003). Diffusion of innovations. Simon and Schuster.

Rogers, E. M., \& Shoemaker, F. F. (1971). Communication of Innovations; A Cross-Cultural Approach.

Semaan, R. W., Lindsay, V., Williams, P., \& Ashill, N. (2019). The influence of gender roles in the drivers of luxury consumption for women: Insights from the gulf region. Journal of Retailing and Consumer Services, 51, 165-175.

Shavinina, L. V. (Ed.). (2003). The international handbook on innovation. Elsevier.

Silva, D. M. L., Prado, P. H. M., Ribeiro, E. B. S., Souza, F. F., \& Korelo, J. C. (2008). Processo de escolha de produtos em constante inovação: inovatividade, objetivos de consumo e percepção da inovação. INMRInnovation \& Management Review, 5(2), 43-56.

Simmel, G. (1904). Fashion. International Quarterly, 10(1), 136.

Slater, D. (2001). Cultura do consumo \& modernidade-Exame. NBL Editora.

Souza, R. V. D. (2002). Adoção de Produtos e Serviços Baseados em Tecnologia pelo Consumidor: uma avaliação da aplicabilidade da Technology Readiness Index no contexto brasileiro.

Strehlau, S., \& Aranha, F. (2004). Adaptação e validação da escala de consumo de status (scs) para uso no contexto brasileiro. Revista de Administração FACES Journal, 3(1).

Tsikriktsis, N. (2004). A technology readiness-based taxonomy of customers: A replication and extension. Journal of Service Research, 7(1), 42-52.

Tushman, M., \& Nadler, D. (1997). Organizando-se para a inovação. STARKEY, K. Como as organizações aprendem. São Paulo: Futura, 445-484. 
